\title{
The Chemically Distinct Nucleus and Structure of the S0 Galaxy NGC 80
}

\author{
O. K. Sil'chenko' ${ }^{1}$, S. E. Koposov', V. V. Vlasyuk ${ }^{2}$, and O. I. Spiridonova ${ }^{2}$ \\ ${ }^{1}$ Sternberg Astronomical Institute, Universitetskiū pr. 13, Moscow, 119992 Russia \\ ${ }^{2}$ Special Astrophysical Observatory, Russian Academy of Sciences, pos. Nizhnǐ Arkhyz, \\ Karachaü-Cherkessia, Russia
}

Received April 3, 2002; in final form, May 23, 2002

\begin{abstract}
The giant lenticular galaxy NGC 80, which is the brightest member of a rich group, possesses a central evolutionarily-distinct region: the stars in the nucleus and in a circumnuclear ring of radius $5^{\prime \prime} t-7{ }^{\prime \prime}$ have a mean age of only $7 \mathrm{Gyr}$, whereas the stellar population of the bulge is older than $10 \mathrm{Gyr}$. The nucleus of NGC 80 is also chemically distinct: it is a factor of $2-2.5$ richer in metals than its immediate neighborhood and is characterized by a high magnesium-to-iron abundance ratio $[\mathrm{Mg} / \mathrm{Fe}] \approx+0.3$. The global stellar disk of NGC 80 has a two-tiered structure: its outer part has an exponential scale length of $11 \mathrm{kpc}$ and normal surface density, while the inner disk, which is also exponential and axisymmetric, is more compact and brighter. Although the two-tiered structure and the chemically distinct nucleus obviously have a common origin and owe their existence to some catastrophic restructuring of the protogalactic gaseous disk, the origin of this remains unclear, since the galaxy lacks any manifestations of perturbed morphology or triaxiality. (C) 2003 MAIK “Nauka/Interperiodica”.
\end{abstract}

\section{INTRODUCTION}

Circumnuclear star-forming rings in galaxies are a spectacular phenomenon and are especially prominent on high-resolution images taken, e.g., by the Hubble Space Telescope (see NGC 4736 in [1] and NGC 7742 in [2] and many other galaxies). The nature of this phenomenon is now believed to be well understood, mainly owing to the efforts of Buta [3, 4]. Since circumnuclear rings are most often found in barred galaxies, it seems likely that the gas that flows along the bar from the outer regions of the disk toward the center accumulates in the region of the inner Lindblad resonance, becoming denser and more compressed, resulting in a burst of star formation. However, rings are also found, albeit less frequently, in galaxies without bars. To explain such cases, the presence of a triaxial halo (NGC 7217 [5]) or transient bars that have disappeared by the time of the observations have been hypothesized [6, 7]. However, it remains unclear whether a circumnuclear ring can develop in an absolutely axisymmetric galaxy; e.g., in a galaxy with regular round isophotes at all galactocentric radii. We have suggested a scenario that does not directly involve the effect of a triaxial potential [8].

When studying chemically peculiar central regions in nearby galaxies, we have come up against the complex structure of isolated compact circumnuclear stellar disks: the stellar populations at the very centers of these disks are often on average 2-3 Gyr older that the populations at the disk edges, $100-400 \mathrm{pc}$ from the center (NGC 1023 [9], NGC 7331 [10]). Moreover, both populations appear to be about 10-12 Gyr younger than the population of the neighboring bulge regions, leading us to conclude that the circumnuclear disks formed during relatively recent bursts of star formation that were efficient and brief (shorter than 1 Gyr) in the core and continued for several Gyr at the periphery of the circumnuclear disk.

Since several gigayears make up an appreciable fraction of the total age of the galaxy and since our estimates suggest that chemically distinct nuclei can be found in 25-50\% of all early-type disk galaxies, a diligent observer has a real chance of catching a galaxy at the time when a burst of star formation has already finished in its nucleus but is ongoing at the periphery of the circumnuclear disk. It is at this time that the galaxy possesses a circumnuclear ring.

The most certain way to distinguish such "relic" rings from rings that form in a quasi-stationary way at the inner Lindblad resonances of bars is that they can be found in round galaxies viewed face-on. We have already had one successful such attempt when we found a ring of HII regions with a radius of just under $1 \mathrm{kpc}$ in the E0 galaxy NGC 759 [11]. This was by no means a blind search: Wilkind et al. [12] had earlier reported the discovery of a rapidly rotating molecular gas torus with a total mass of $2.4 \times 10^{9} M_{\odot}$ in this elliptical galaxy, which by itself is a unique phenomenon. We used two-dimensional spectroscopy and surface photometry to analyze the structure of 
the stellar component and verified that the massive, extended, spheroidal subsystem contains a compact stellar circumnuclear disk embedded in it, where active star formation is ongoing at a considerable galactocentric distance. We thus caught a chemically distinct central stellar system at the very time of its formation in NGC 759.

In the current paper, we report a second discovery of a ring of young stars in an early-type round galaxy. This discovery was almost accidental, except for the fact that we had listed the galaxy as a candidate for a galaxy possessing a chemically distinct nucleus [13]. Here, we analyze our results for the giant lenticular galaxy NGC 80.

The main global parameters of the galaxy are listed in Table 1. It is the brightest member of either a rich group (the RC1 catalog) or a poor cluster (the UGC catalog): the aggregate contains nine NGC galaxies brighter than $16^{m}\left(M_{B}<-18.5\right)$ of various morphological types within several arcmin, or half a megaparsec, of each other. NGC 80 is not located at the geometric center of the group but somewhat to the side. This lenticular galaxy does not exhibit peculiarities of any kind: it has a very red integrated color, as expected for a bright, early-type galaxy (Table 1, see also [14]), no emission lines in the spectrum of its nucleus, and no detected neutral hydrogen [15]. The galaxy has virtually perfectly round isophotes, and attempts to measure its rotation have yielded an upper limit of $v_{\text {los,max }}<15 \mathrm{~km} / \mathrm{s}$ for the line-of-sight projection of the rotational velocity [16], indicating that the galaxy appears symmetric and must be viewed face-on. We have performed both two-dimensional spectroscopy and surface photometry of NGC 80 , and below we report results that improve our understanding of this interesting object.

\section{OBSERVATIONS AND DATA REDUCTION}

We observed the central part of NGC 80 on August 17, 1996, with the Multi-Pupil Field Spectrograph (MPFS) mounted at the primary focus of the 6$m$ telescope of the Special Astrophysical Observatory (SAO) of the Russian Academy of Sciences (see [17] for a description of the instrument). Our observations covered the spectral region of the MgIb $\lambda 5175$ absorption line (wavelength interval $4880-5650 \AA$ ) with a reciprocal dispersion of $1.6 \AA$ pixel (a spectral resolution of about $4 \AA$ ) and a seeing equal to $2.5^{\prime \prime}$. We observed the object over a one-hour exposure time and then obtained a 20 min exposure of an "empty" area of sky to be subtracted from the integrated object frame after normalization and smoothing. The detector used was a $520 \times 580$ CCD manufactured by the Russian company Elektron. During the MPFS observations,
Table 1. Global parameters of NGC 80

\begin{tabular}{l|l}
\hline \multicolumn{1}{c|}{$\begin{array}{c}\text { Morphological } \\
\text { type (NED) }\end{array}$} & \multicolumn{1}{c}{$\mathrm{SAO}$} \\
\hline$R_{25}$ & $21 \mathrm{kpc}$ \\
$B_{T}^{0}(\mathrm{RC} 3)$ & 13.03 \\
$M_{B}$ & -21.43 \\
$(B-V)_{T}^{0}(\mathrm{RC} 3)$ & 0.94 \\
$(U-B)_{T}^{0}(\mathrm{RC} 3)$ & 0.64 \\
$V_{r}(\mathrm{NED})$ & $5698 \mathrm{~km} \mathrm{~s}^{-1}$ \\
Distance (LEDA) & $\left.78 \mathrm{MnK} \mathrm{km} \mathrm{s}^{-1} \mathrm{Mpc}^{-1}\right)$ \\
& $\left(H_{0}=75 \mathrm{~km}\right.$ \\
Inclination (LEDA) & $28^{\circ}$ \\
PA $_{\text {phot }}$ & $?$ \\
\hline
\end{tabular}

an $8 \times 12$ array of microlenses constructs a matrix of pupils that is used as the input for the diffraction spectrograph. This configuration enables up to 96 spectra to be recorded simultaneously, each corresponding to a spatial element of the galaxy image. In our case, each element had a size of $1.6^{\prime \prime} \times 1.6^{\prime \prime}$. We calibrated the wavelength scale using a separate exposure of a $\mathrm{He}-\mathrm{Ne}-\mathrm{Ar}$ lamp spectrum and corrected the spectra for vignetting and the different transmissions of the microlenses using a spectrum of the morning sky. The main stages of the data reduction-bias subtraction, cosmic-ray removal, extraction of the onedimensional spectra from the frame, and construction of the two-dimensional surface-brightness distributions and velocity fields-were carried out using a software package developed at the SAO [18].

We used the MPFS observations at 4880-5650 to analyze the radial dependences of the equivalent widths of absorption lines, and also to construct the two-dimensional stellar radial-velocity field at the center of the galaxy. We achieved the first task by summing spectra within concentric rings centered on the nucleus of the galaxy with widths and a radius step of 1.6 arcsec; i.e., equal to the size of a spatial element. This method enabled us to maintain an approximately constant signal-to-noise ratio as the radius increased, which is impossible, for example, in long-slit observations. We then computed the $H \beta$, Mgb, Fe 5270, and Fe 5335 line indices in the well-known Lick system [19]. Detailed model computations in the context of models of old stellar populations are available for these strong absorption lines [20, 21].

To construct the two-dimensional stellar radialvelocity field at the center of the galaxy, we crosscorrelated the spectrum of each spatial element with 
Table 2. Photometric observations of NGC 80

\begin{tabular}{c|l|c|c|l|l}
\hline Date & Telescope & Filter & $\begin{array}{c}\text { Exposure } \\
\text { time, min }\end{array}$ & Seeing, arcsec & $\begin{array}{c}\text { Scale, } \\
\text { arcsec/pixel }\end{array}$ \\
\hline Nov. 29, 1997 & $1.2 \mathrm{~m}$, OHP & $B$ & 10 & 3.1 & 0.69 \\
Nov. 29, 1997 & $1.2 \mathrm{~m}, \mathrm{OHP}$ & $V$ & 5 & 2.8 & 0.69 \\
Nov. 29, 1997 & $1.2 \mathrm{~m}, \mathrm{OHP}$ & $R$ & 5 & 2.5 & 0.69 \\
July 18, 2001 & $1 \mathrm{~m}, \mathrm{SAO}$ & $V$ & 30 & $1.9 \times 2.3$ & $0.27 \times 0.37$ \\
July 18, 2001 & $1 \mathrm{~m}, \mathrm{SAO}$ & $R$ & 35 & $1.8 \times 2.2$ & $0.27 \times 0.37$ \\
Aug. 16, 2001 & $1 \mathrm{~m}, \mathrm{SAO}$ & $B$ & 20 & 3.2 & $0.27 \times 0.37$ \\
Aug. 16, 2001 & $1 \mathrm{~m}, \mathrm{SAO}$ & $V$ & 10 & 2.9 & $0.27 \times 0.37$ \\
Aug. 16, 2001 & $1 \mathrm{~m}, \mathrm{SAO}$ & $R$ & 10 & 2.6 & $0.27 \times 0.37$ \\
Aug. 16, 2001 & $1 \mathrm{~m}, \mathrm{SAO}$ & $I$ & 15 & 2.8 & $0.27 \times 0.37$ \\
Aug. 17, 2001 & $1 \mathrm{~m}, \mathrm{SAO}$ & $B$ & 20 & 2.2 & $0.27 \times 0.37$ \\
Aug. 17, 2001 & $1 \mathrm{~m}, \mathrm{SAO}$ & $V$ & 10 & 2.0 & $0.27 \times 0.37$ \\
Aug. 17, 2001 & $1 \mathrm{~m}, \mathrm{SAO}$ & $R$ & 10 & 1.8 & $0.27 \times 0.37$ \\
Aug. 17, 2001 & $1 \mathrm{~m}, \mathrm{SAO}$ & $I$ & 10 & 1.8 & 0.37 \\
\hline
\end{tabular}

the spectra of $\mathrm{K} 0-\mathrm{K} 2$ giants observed on the same night and with the same instruments as the galaxy, after subtracting the continuum and converting the results to a velocity scale. We monitored the accuracy of the wavelength scale and the zero point of the mea-

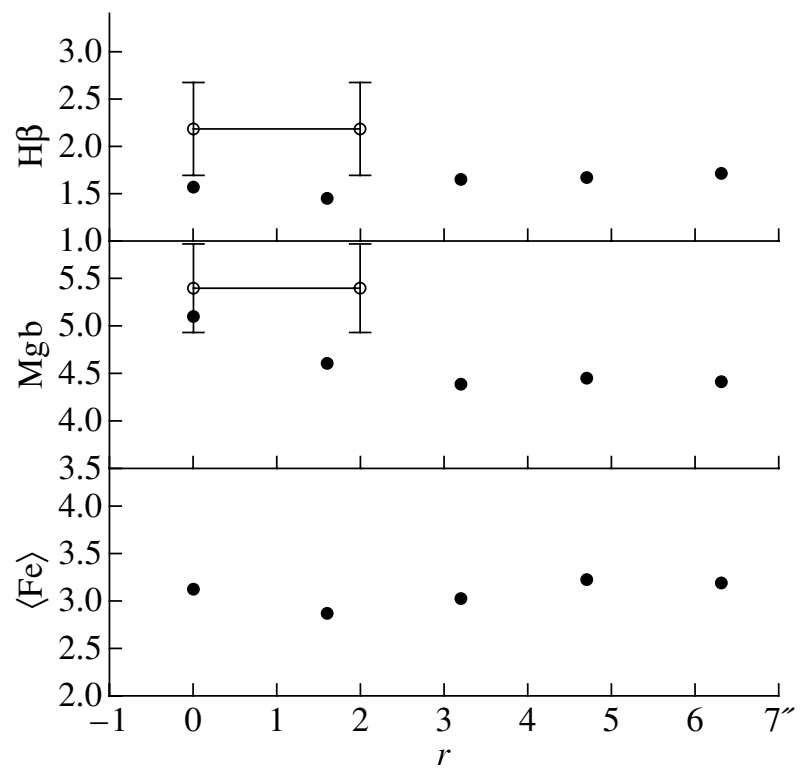

Fig. 1. Radial variations of the azimuthally averaged measurements of the $\mathrm{H} \beta, \mathrm{Mgb}$, and $\langle\mathrm{Fe}\rangle \equiv(\mathrm{Fe} 5270+$ Fe5335)/2 absorption-line indices in NGC 80 based on our data (filled circles). For comparison, we have also plotted the measurements of Trager et al. [24] made in a $2^{\prime \prime} \times 4^{\prime \prime}$ aperture (open circles with error bars). sured velocities using observations of the $\lambda 5577 \AA$ night-sky line. We estimate the accuracy of individual stellar radial-velocity measurements to be $20 \mathrm{~km} / \mathrm{s}$ and the accuracy of the derived equivalent widths in the azimuthally averaged spectra to be $0.1 \AA$. In this paper, we will discuss only the properties of the stellar population. Our analysis of the existing data indicated that the kinematic properties of NGC 80 require further study.

We conducted photometric observations of NGC 80 in July-August 2001 with the 1-m Zeiss telescope of the SAO. The detector used was a $520 \times 580$ Russian-made Elektron CCD with $18 \times 24 \mu \mathrm{m}$ rectangular pixels. After reduction to square pixels, we obtained a $397 \times 580$ image with a scale of $0.37^{\prime \prime}$ per pixel. We calibrated the magnitudes to the standard Johnson-Cousins system by comparing our data with the aperture photometry of Poulain [22]; we also observed Landolt [23] photometric standards on one of the nights (August 17, 2001 ). Table 2 gives a detailed log of the observations. In addition to our own data, we used FITS data taken from the HYPERCAT archive of extragalactic data. These photometric data were obtained at the $1.2-\mathrm{m}$ telescope of the Observatoire de Haute-Provence (OHP) under very modest seeing conditions (about $3^{\prime \prime}$ ) and with a rather coarse scale of $0.7^{\prime \prime} /$ pixel, but they are deeper than our photometry in terms of limiting magnitude. 

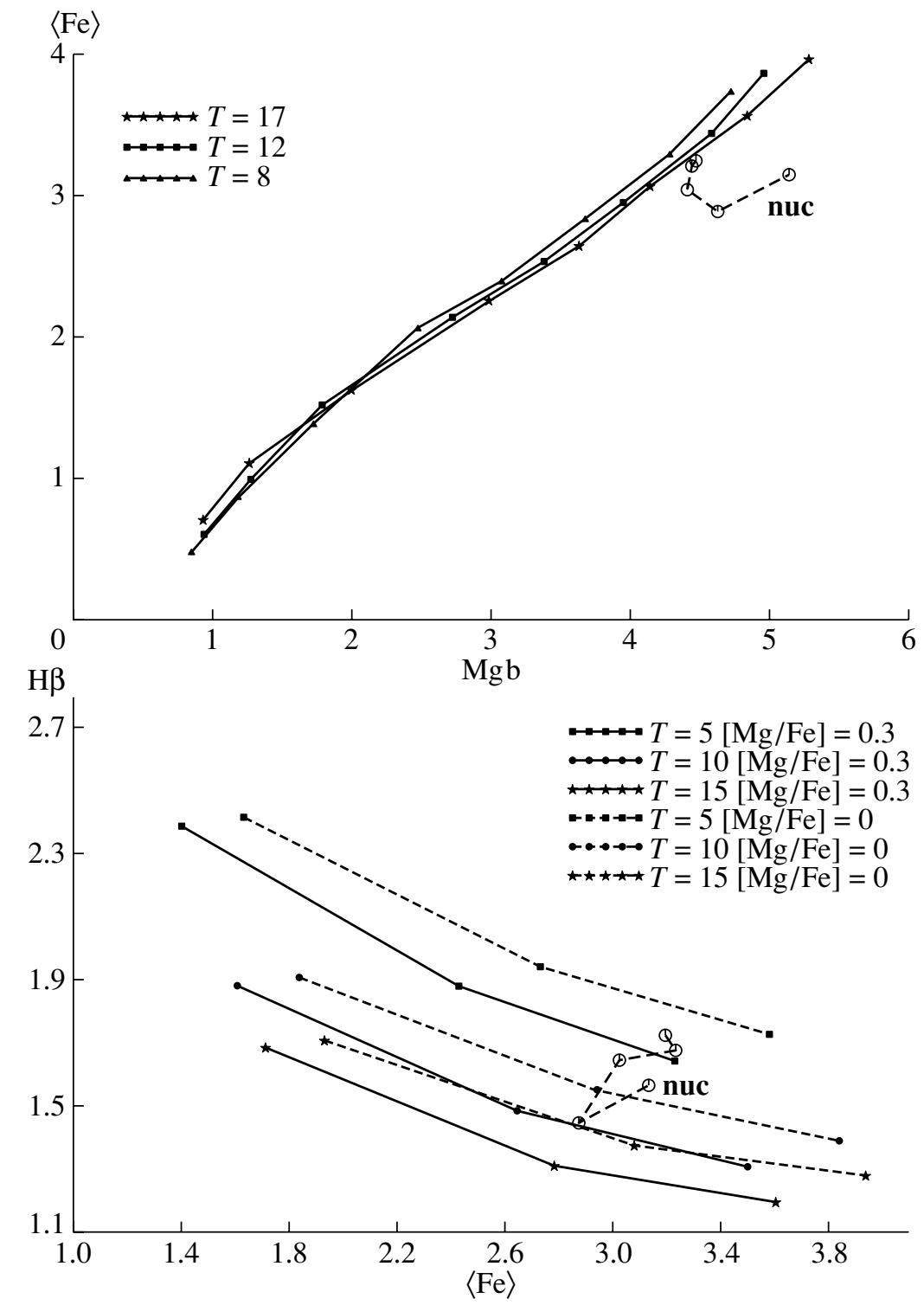

Fig. 2. Index-index diagnostic diagrams for the azimuthally averaged measurements for NGC 80 (large open circles). The MPFS data are taken with a step of $1.6^{\prime \prime}$ in galactocentric radius and are connected by the dashed line in order of increasing $R$. The nucleus is indicated as "nuc." The small symbols connected by solid lines (or by solid lines for $[\mathrm{Mg} / \mathrm{Fe}]=+0.3$ and dashed lines for $[\mathrm{Mg} / \mathrm{Fe}]=0$ in the lower diagram) represent models for stellar populations of the same age adopted from [20] (upper) and [21] (lower). The age $T$ of each model is indicated in Gyr. The model metallicities corresponding to the sequence of small symbols connected by lines are (from right to left): $+0.50,+0.25,0.00,-0.22,-0.50,-1.00,-1.50$, and -2.00 for models [20] and $+0.4,0.0$, and -0.7 for models [21].

\section{THE CHEMICALLY DISTINCT NUCLEUS AND "YOUNG" RING AT THE CENTER OF NGC 80}

Figure 1 shows the radial variation of the Lick indices measured for the integrated spectra of annular rings in the radius interval $0^{\prime \prime}-7^{\prime \prime}$. We can see that the iron index $\langle\mathrm{Fe}\rangle$ remains virtually constant with radius and the observed fluctuations within $2.9-3.2 \AA$ confirm the $0.1 \AA$ accuracy of the individual estimates. However, the magnesium index exhibits a prominent
$0.7 \AA$ discontinuity nearly coincident with the unresolved nucleus of the galaxy. Beyond the nucleus (whose effect in the case of $2.5^{\prime \prime}$ seeing becomes negligible at $R \geq 3^{\prime \prime}$ ), the radial profile of the magnesium index flattens, and becomes fairly constant in the "bulge." Thus, Fig. 1 leads us to conclude that the nucleus of NGC 80 is chemically distinct from the bulge. If interpreted in terms of the models for old stellar populations suggested by Worthey [20], a $0.7 \AA$ 
discontinuity in the Mgb index corresponds to about a twofold variation of the mean stellar metallicity.

However, the variations of a single index are degenerate with respect to the effects of the age and metallicity of the stellar population: a decrease in either parameter results in a decrease in the equivalent widths of metal absorption lines and an increase in the equivalent widths of hydrogen absorption lines (see, e.g., [25]). Therefore, to remove this degeneracy and determine both characteristics simultaneously, we must compare the observational data with models for the integrated spectra of stellar populations in "index-index diagrams." In this case, the above estimate of the metallicity variation corresponding to $\Delta M g b=0.7 \AA$ is valid only if the stellar populations in the nucleus and bulge have the same mean ages.

We will first estimate the magnesium-to-iron abundance ratio. This is necessary in order to correctly choose a system of models for the age estimation. Figure $2 \mathrm{a}$ shows a $(\langle\mathrm{Fe}\rangle, \mathrm{Mgb})$ diagram comparing our data with Worthey's [20] models for the solar magnesium-to-iron abundance ratio $[\mathrm{Mg} / \mathrm{Fe}]=0$. On this diagram, model isochrones along which the metallicity $[\mathrm{Fe} / \mathrm{H}]$ varies from -2 to +0.5 form a narrow beam, and it is immediately apparent that the nucleus of NGC 80 deviates significantly to the right of the model band, which includes various stellar populations with $[\mathrm{Mg} / \mathrm{Fe}]=0$. Most of the elliptical galaxies behave in the same way [26], which is indicative of magnesium (and other $\alpha$ process elements) being overabundant relative to iron. Modern concepts about the chemical evolution of galaxies explain this as being due to the brevity of the star-formation epoch in the galaxy, which lasts less than 1 Gyr. Calibration of the displacement of the nucleus of NGC 80 in Fig. 2 relative to the model sequences $[\mathrm{Mg} / \mathrm{Fe}]=0$ based on the computations of Tantalo et al. [21] yields $[\mathrm{Mg} / \mathrm{Fe}] \approx+0.3$. The $\mathrm{Mg} / \mathrm{Fe}$ ratio decreases with galactocentric distance and is close to the solar value in the bulge, at $R \geqslant 3^{\prime \prime}$.

Figure $2 \mathrm{~b}$ is a diagnostic diagram for determining the ages of stellar populations: we compare our data for NGC 80 with the models of Tantalo et al. [21] in the $(\mathrm{H} \beta,\langle\mathrm{Fe}\rangle)$ plane. Although Tantalo et al. [21] did not model the Mgb index, their models are computed for three different $[\mathrm{Mg} / \mathrm{Fe}]$ ratios, whereas Worthey [20] modeled about 20 different indices for only the solar abundance ratio. We used the models of Tantalo et al. [21] for $[\mathrm{Mg} / \mathrm{Fe}]=0$ and $[\mathrm{Mg} / \mathrm{Fe}]=$ +0.3 for the bulge and nucleus, respectively. It is immediately apparent from Fig. $2 \mathrm{~b}$ that, if we ignored the difference between the $\mathrm{Mg} / \mathrm{Fe}$ ratios in the nucleus and bulge, the systematic error in the age estimates would amount to $2-3$ Gyr for both populations. At the same time, if correctly computed, the mean age for stars in the nucleus is $7 \mathrm{Gyr}$; the mean stellar age increases abruptly in the immediate vicinity of the nucleus and then drops again to 7 Gyr at a galactocentric radius of $5^{\prime \prime}-7^{\prime \prime}$. We cannot reliably determine the age of the stellar population in the "old" region at $R \approx 2^{\prime \prime}$, since these measurements are too contaminated by the nucleus at our seeing. However, this population is obviously older than 10 Gyr. Thus, in a lenticular galaxy that is absolutely devoid of gas and star formation, we have found a stellar nucleus and a ring with a radius of about $6^{\prime \prime}$, both with a mean age of 7 Gyr. This is significantly lower than the mean age of the bulges of lenticular galaxies, which exceeds 10 Gyr [27]. Since the nucleus of NGC 80 is chemically distinct, these results obviously suggest that we have found traces of a secondary burst of star formation that had a fairly complex geometry.

\section{RESULTS OF OUR PHOTOMETRIC ANALYSIS OF NGC 80}

We already mentioned in Section 2 that the data from the Observatoire de Haute-Provence have lower spatial resolution than our observations of August 17, 2001 , but are deeper in terms of their limiting magnitude. We accordingly used the OHP $B V R$ images to investigate the large-scale structure of the galaxy and our own $B V R I$ images of August 17 to analyze the circumnuclear region.

Figure 3a shows the results of an isophotal analysis of the OHP data: radial variations of the position angle of the isophotal major axis and ellipticity. The ellipticity is small throughout the region studied, as is expected from the round appearance of NGC 80; however, it is not equal to zero. The LEDA database (Table 1) also gives $i=28^{\circ}$ and not $0^{\circ}$; i.e., the galaxy is not viewed exactly faceon. Therefore, the asymptotic value $(1-b / a)_{0}=0.09$ obviously characterizes the inclination of the galactic disk to the line of sight - the galaxy is viewed at an angle of $25^{\circ}$. However, it is then surprising that the ellipticity reaches the asymptotic level at a galactocentric radius as small as $\sim 10^{\prime \prime}$; this may imply that the photometric structure of the giant lenticular galaxy NGC 80 is already dominated by the disk at $R \approx 10^{\prime \prime}$, since the axisymmetric bulge, being "plump" in the direction of the vertical coordinate, always yields a lower projected ellipticity than the disk. At radii of $30^{\prime \prime}-45^{\prime \prime}$, the ellipticity begins to exhibit some irregularity in its behavior; however, the radial variations settle down in the vicinity of $1-b / a \approx 0.1$ farther from the center. The position angle of the isophotal major axis also behaves fairly monotonically and does not deviate from $\mathrm{PA}_{0} \approx 180^{\circ}$ by more than $5^{\circ}$ virtually throughout the radius interval studied. On the whole, 


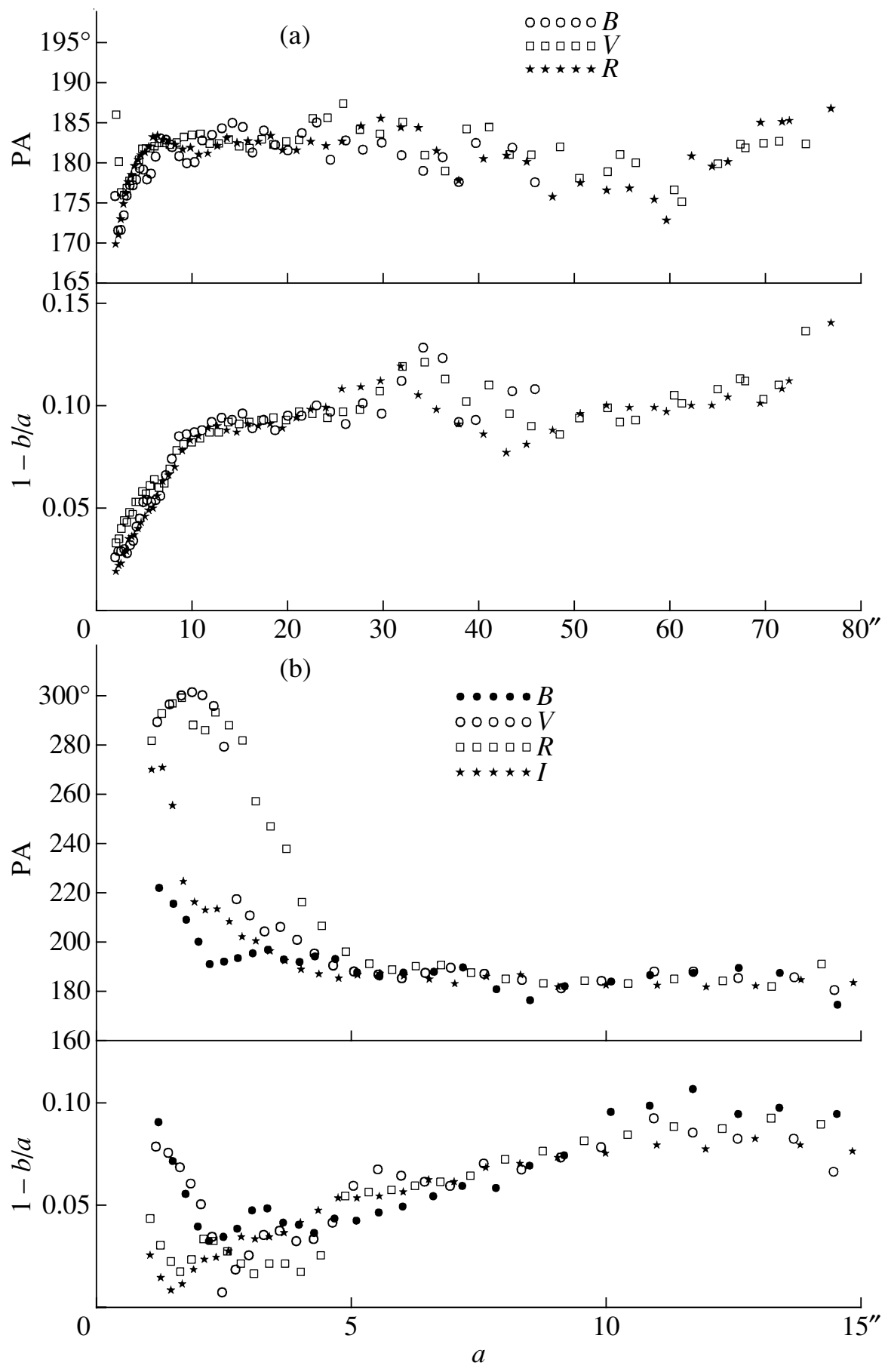

Fig. 3. Radial variations of the isophotal parameters (the position angle of the line of nodes and isophotal ellipticity) in NGC 80 in various photometric bands for (a) data obtained at the 1.2-m OHP telescope and (b) our own observations of August 17, 2001 with the 1-m SAO telescope. The spatial resolutions are $3^{\prime \prime}$ and better than $2^{\prime \prime}$ in (a) and (b), respectively.

the dependences shown in Fig. 3a are typical of a galaxy dominated exclusively by a flat, round stellar disk with no warps or bar.

Figure $3 \mathrm{~b}$ is similar to Fig. 3a but is based on our data of August 17, 2001, and shows only the very central part of NGC 80 . Recall that our $B$ image has a spatial resolution of $2.2^{\prime \prime}$, whereas the resolutions in the remaining three filters are better than $2^{\prime \prime}$. This seemingly small improvement in the spatial resolution had a very dramatic effect on the results of the isophotal analysis: the isophotal major axis rotated by about $90^{\circ}$ at the center, and, after reaching a minimum of 0.03 at $R=2.5^{\prime \prime}$, the ellipticity again increases toward the nucleus, reaching $0.08-0.09$ in the blue 


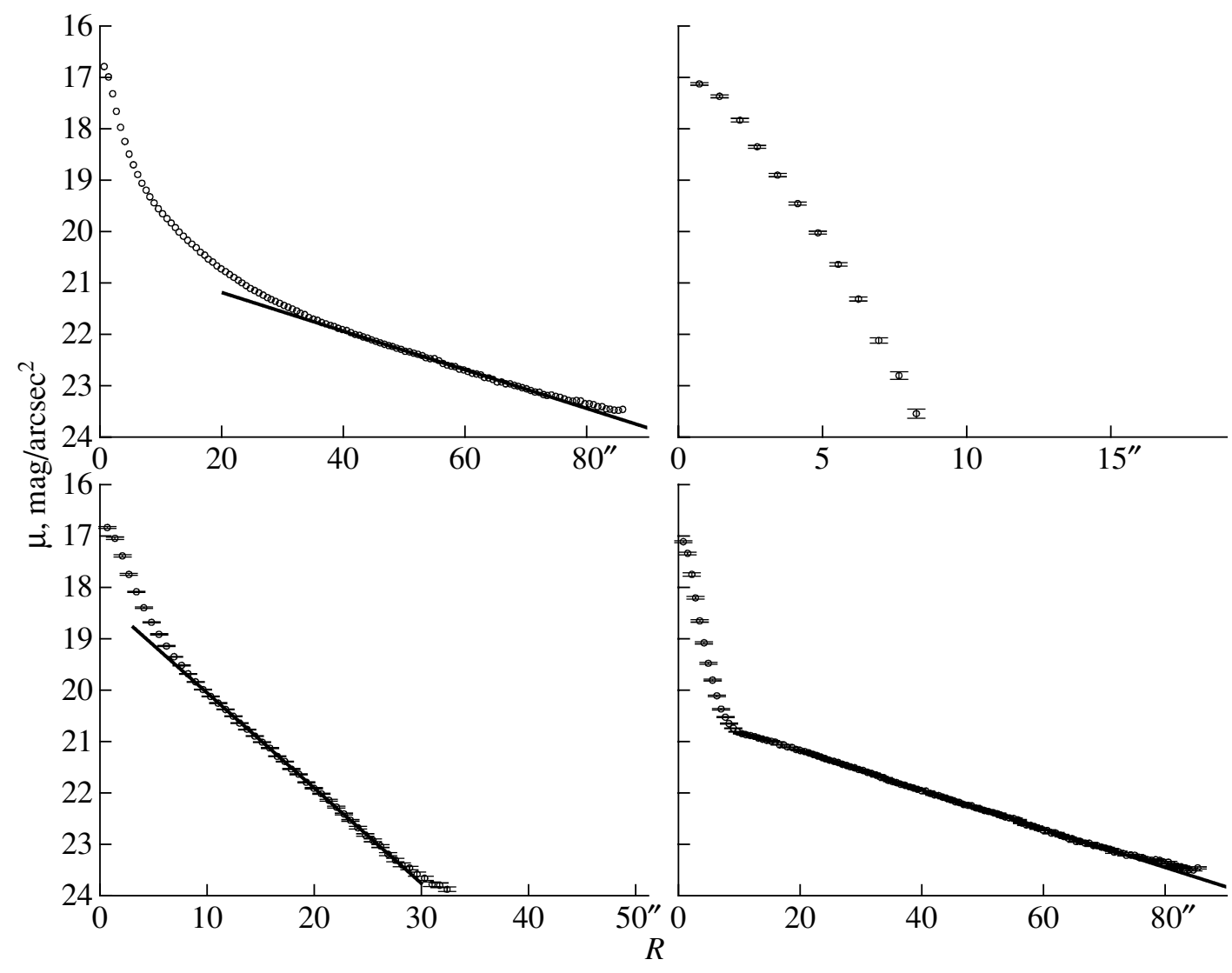

Fig. 4. Example of a decomposition of the azimuthally averaged $R$ surface-brightness profile. Top left: the initial profile (OHP data) with its outermost part fitted by an exponential law; bottom left: the residual brightness profile after subtracting the outer disk, with its outermost part fitted by an exponential law; bottom right: the residual brightness profile after subtracting the inner disk from the initial image (the outer disk obeys a single exponential law throughout the radius interval $8^{\prime \prime}-80^{\prime \prime}$ ); top right: the residual brightness profile after subtracting two exponential disks from the initial image.

filters $(B, V)$. All these peculiarities in the behavior of the isophotes are concentrated near the center at $R \leq 3^{\prime \prime}$, which is close to the limit of our spatial resolution and so may not represent entirely trustworthy measurements. However, our results are supported by those of other studies. The measurements of a $V$ photographic image of NGC 80 by Magrelli et al. [28] also showed the isophotes at the center of the galaxy to rotate by $90^{\circ}$ and the ellipticity of the isophotes to reach 0.15 near the nucleus. Thus, the chemically distinct nucleus of NGC 80 also stands out as a separate structural subsystem of the galaxy.

Let us now analyze the radial surface-brightness profile (Fig. 4 shows the $R$ profile as an example). In contrast to the dependences shown in Fig. 3a, the brightness profile changes its behavior near $R \approx 35^{\prime \prime}-40^{\prime \prime}:$ the galaxy appears to consist of several global structural components. Let us first assume, in line with tradition, that the outer and inner components are an exponential disk and de Vaucouleurs bulge, respectively. The $B$ brightness profile in the radius interval $R \approx 10^{\prime \prime}-46^{\prime \prime}$ can indeed be fitted within the observational errors by a $R^{1 / 4}$ law; this implies that the global exponential disk does not have an inner part $R<40^{\prime \prime}$; however, global disks with central "holes" are quite common in earlytype disk galaxies [29, 30]. However, this approach leads to rather unusual bulge parameters: $r_{e}=36^{\prime \prime}$, or $13.6 \mathrm{kpc}$ and $B_{e, 0}=24.14$, which deviates by more than $1^{m}$ (toward lower brightnesses) from the well-known statistical dependence of Kormendy [31], $B_{e, 0}=19.74+3.02 \log r_{e}$. The implied exponential scale length of the outer disk is $11 \mathrm{kpc}$, which is unusually large, and is smaller than the effective bulge radius, although, statistically, it should be the other way round [32]. The situation is clarified if we approach the problem from a different angle and abandon the hypothesis of a central "hole" in the outer disk. In this case, the residual brightness profile after subtracting the modeled outer exponential disk from the observed image of NGC 80 can likewise be fitted by an exponential law in the radius interval $R \approx 8^{\prime \prime}-28^{\prime \prime}$ (Fig. 4) but, naturally, with a shorter scale length (of about $2 \mathrm{kpc}$ ) and higher central 
Table 3. Parameters of exponential fits to the photometric components of NGC 80

\begin{tabular}{l|c|r|c|c|c}
\hline Component (disk) & $\mu_{0}, \mathrm{mag} / \operatorname{arcsec}^{2}(2 \mathrm{D})$ & $r_{0}^{\prime \prime}$ & $\mu_{0}, \mathrm{mag} / \mathrm{arcsec}^{2}$ & $r_{0}^{\prime \prime}$ & $r_{0}, \mathrm{kpc}$ \\
\hline \multicolumn{6}{c}{$B$} \\
\hline Outer $\left(40^{\prime \prime}-75^{\prime \prime}\right)$ & 21.7 & 27.3 & $21.82 \pm 0.01$ & $28.7 \pm 0.2$ & 10.8 \\
Inner $\left(10^{\prime \prime}-24^{\prime \prime}\right)$ & 19.5 & 5.6 & $19.66 \pm 0.02$ & $5.91 \pm 0.04$ & 2.2 \\
\hline \multicolumn{6}{l}{$V$} \\
\hline Outer $\left(40^{\prime \prime}-77^{\prime \prime}\right)$ & 20.8 & 28.3 & $20.90 \pm 0.01$ & $29.4 \pm 0.1$ & 11.1 \\
Inner $\left(8^{\prime \prime}-27^{\prime \prime}\right)$ & 18.5 & 5.7 & $18.63 \pm 0.01$ & $6.01 \pm 0.02$ & 2.3 \\
\hline \multicolumn{5}{|c}{$R$} \\
\hline Outer $\left(40^{\prime \prime}-77^{\prime \prime}\right)$ & 20.1 & 27.3 & $20.28 \pm 0.01$ & $28.8 \pm 0.1$ & 10.9 \\
Inner $\left(8^{\prime \prime}-27^{\prime \prime}\right)$ & 17.9 & 5.5 & $18.04 \pm 0.01$ & $5.84 \pm 0.01$ & 2.2 \\
\hline
\end{tabular}

brightness. The exponential law in the inner component can be followed over three scale lengths, giving the fit very high confidence. After subtracting the modeled inner disk from the observed galaxy image, it becomes immediately apparent that the outer disk exhibits a rarely seen exponential behavior over the radius interval $R \approx 10^{\prime \prime}-80^{\prime \prime}$ (Fig. 4). This "residual" image enables us to refine the parameters of the outer disk, which we have done.

Table 3 lists the parameters for our approximations of the NGC 80 images using two exponential laws in all three $B V R$ filters and the OHP data. We present our results for two methods: iterative decomposition of the azimuthally averaged brightness profiles and fitting the two-dimensional images by a sum of two exponential disks. The results obtained using the two methods are in good agreement. The central surface densities have been corrected for Galactic absorption in accordance with the recommendations given in the NED database.

It is now clear that the main structural components of NGC 80 are two exponential stellar disks with different scale lengths. The outer disk has fairly ordinary characteristics, although its scale length is among the longest known. Its central brightness is absolutely typical of such objects and lies at the center of the well-known distribution of Freeman [33]. The inner disk has an unusually high central brightness but is a flat disk and not a so-called exponential bulge, since, as we pointed out above, the isophotal ellipticity already reaches its asymptotic level at $R \approx 10^{\prime \prime}$. After subtracting two asymptotic disks from the images of NGC 80, we obtain a compact, circumnuclear component with a radius of about $R \approx 7^{\prime \prime}-8^{\prime \prime}$ and a weakly convex brightness profile (Fig. 4). However, the poor seeing of the OHP images makes it impossible to analyze the profile of this compact component, so its nature remains unclear.
Finally, Fig. 5 shows azimuthally averaged radial color profiles for NGC 80 based on our August 2001 data, for which the calibration in the standard Johnson-Cousins system is most reliable. Out to $R=10^{\prime \prime}-20^{\prime \prime}$, we use the data for August 17, which have the best spatial resolution, and farther from the center, we use coadded data for August 16 and 17. All the color profiles exhibit a well-defined "blue" ring at $R=7^{\prime \prime}-8^{\prime \prime}$ and, although our spectroscopic data end at $R=6.3^{\prime \prime}$, the blue ring in Fig. 5 can nevertheless be identified with the region with a relatively young stellar population in Fig. 2. This region apparently ends completely by $R \approx 12^{\prime \prime}$. The color difference between the nucleus and the ring can be interpreted as reflecting the difference in the mean metallicities of the corresponding stellar populations. In this case, $\Delta(B-V)=0.12$ corresponds to $\Delta[\mathrm{m} / \mathrm{H}]=+0.4$; i.e., the nucleus is richer in metals (here, we have adopted the color calibration in terms of properties of stellar populations of [34]). Recall that the magnesium line yielded $\Delta[\mathrm{m} / \mathrm{H}]=+0.3$. The color difference between the ring and more outer regions at $R>12^{\prime \prime}$ must be due to a superposition of the effects of age and metallicity differences, which cannot be disentangled without spectroscopic data. The color variations in NGC 80 are fairly small outside the circumnuclear region. More precisely, we can say with confidence that the color does not change at all with radius within the inner disk, while there may be a color difference between the inner and outer disks (see the $B-I$ and $V-I$ profiles in Fig. 5), with the outer disk being slightly bluer than the inner disk.

\section{RESULTS AND DISCUSSION}

We find the giant lenticular galaxy NGC 80, which is the brightest member of a rich group (or a poor cluster), to possess a chemically distinct nucleus: the 


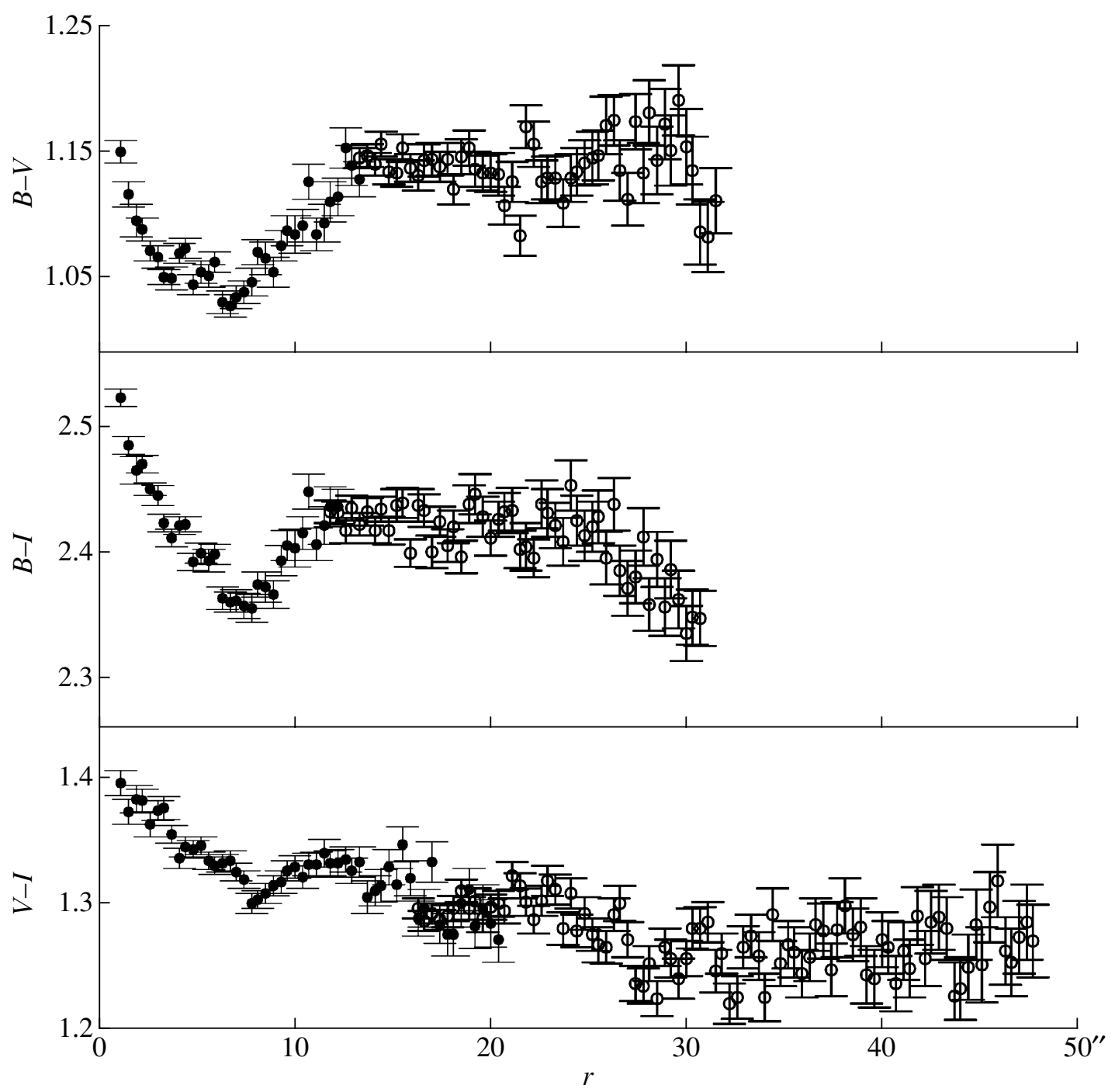

Fig. 5. Azimuthally averaged color profiles for NGC 80 derived from the 1-m SAO data. The filled and open circles show the high-resolution data of August 17, 2001, and the coadded data of August 16 + 17, 2001, respectively.

mean metallicities of the nucleus and circumnuclear region $\left(R=2^{\prime \prime}-7^{\prime \prime}\right)$ differ by about +0.3 to +0.4 dex, or a factor of $2-2.5$. The age structure of the central region of $\mathrm{NGC} 80$ is also complex: the mean age of the populations in the nucleus and in the ring of radius $R=5^{\prime \prime}-8^{\prime \prime}$ is $5-7$ Gyr, whereas the stars located between the nucleus and this ring are much older. It appears that there was a secondary burst of star formation that produced the chemically distinct nucleus, which had the geometrical form of a ring of radius 2-3 kpc and an additional source of star formation at the center of this ring. The fact that the elemental abundance ratio in the nucleus is $[\mathrm{Mg} / \mathrm{Fe}] \approx+0.3$ but is $[\mathrm{Mg} / \mathrm{Fe}] \approx 0$ in the ring leads us to conclude that the burst of star formation was shorter (albeit more efficient) in the nucleus than in the ring. Given the equal mean ages of the stellar populations, this implies that the onset of the burst of star formation in the ring preceded that in the nucleus. At the same time, triggering a ring-shaped circumnuclear burst of star formation requires a bar (triaxial potential), which is currently absent from NGC 80.

The global structure of NGC 80 is also unusual. The disk in this galaxy has a sort of two-tiered structure consisting of an outer exponential disk with a long scale length and normal central surface brightness and an inner, compact, bright disk that is also exponential. Since the galaxy is viewed almost faceon, we can firmly conclude that the two disks are round, flat, and coplanar. We have already encountered two-tiered disks in galaxies with chemically distinct nuclei and suggested that these two phenomena may be related. If at some time an outer tidal action or internal bar instability perturbed the global gaseous 
disk of a spiral galaxy, the infall of gas from the outer regions of the galaxy into its inner regions should have triggered both a central burst of star formation and an abrupt change in the characteristic radial scale length for the distribution of newly born stars. However, in both NGC 615 [35] and NGC 7217 [36], to which this hypothesis was to be applied, the inner compact disks have oval shapes, which is clear evidence of the past existence of a global bar, which could have triggered the large-scale redistribution of gas in the disk.

In two other galaxies (the lenticulars NGC 4429 and NGC 7013), we [37] found relatively young circumnuclear rings, similar to the ring in NGC 80 but with smaller radii of about $0.5 \mathrm{kpc}$. However, the global brightness profiles of these galaxies contain an additional component-a "lens," which is also oval and, again, is believed to have been produced by the disruption of a bar. In NGC 80, we have found for the first time a ring structure for a central burst of star formation and a multi-tiered structure for the stellar disk combined with clear axial symmetry of all the galactic components. Is it possible in a very dense environment with a high probability for multiple impacts of satellites into the galaxy to obtain a pure $m=0$ mode for the perturbation of the global gaseous disk? Or did the galaxy actually have a bar, which has dissipated without leaving a trace? Only specialized three-dimensional models can provide answers to these questions. Resolving these problems may also shed light on the origin of lenticular galaxies: it is possible that NGC 80 was previously a giant latetype spiral galaxy that lost its gas as a result of catastrophic events whose consequences we have just discovered. In any case, NGC 80 lacks any significant spheroidal component (bulge), which is believed to be a mandatory attribute of a classical lenticular galaxy.

\section{ACKNOWLEDGMENTS}

We are grateful to V.L. Afanasiev, A.N. Burenkov, and S.N. Dodonov of the Special Astrophysical Observatory for their support with the observations at the 6-m telescope and to V.P. Mikhailov for his support with the observations at the 1-m telescope. Some of the observational data used in this work were obtained on the 6-m telescope funded by the Ministry of Industry and Science of the Russian Federation (registration number 01-43). We also used photometric data obtained by Philippe Prugnel with the 1.2-m telescope of the Observatoire de Haute-Provence. We acquired these data from the HYPERCAT archive of extragalactic data. We also made use of the LyonMeudon extragalactic database (LEDA), provided by the LEDA team at the CRAL observatory (France), and the NASA/IPAC NED extragalactic database, operated by the Jet Propulsion Laboratory of the
California Institute of Technology under a contract with the National Aeronautics and Space Administration (USA). This work was supported by the Russian Foundation for Basic Research (project no. 0102-16767) and the Federal Research and Technology Program "Astronomy. Fundamental Space Research" (section "Astronomy") 1.2.4.1.

\section{REFERENCES}

1. D. Maoz, A. V. Filippenko, L. C. Ho, et al., Astrophys. J., Suppl. Ser. 107, 215 (1996).

2. P. T. de Zeeuw, M. Bureau, E. Emsellem, et al., Mon. Not. R. Astron. Soc. 329, 513 (2002).

3. R. Buta, Publ. Astron. Soc. Pac. 105, 654 (1993).

4. R. Buta and D. A. Crocker, Astron. J. 105, 1344 (1993).

5. R. Buta, W. van Driel, J. Braine, et al., Astrophys. J. 450, 593 (1995).

6. R. Buta, Astrophys. J. 370, 130 (1991).

7. L. Athanassoula, Barred Galaxies, Ed. by R. Buta, D. A. Crocker, and B. G. Elmegreen, Astron. Soc. Pac. Conf. Ser. 91, 309 (1996).

8. O. K. Sil'chenko, Astron. Astrophys. Trans. 20, 123 (2001).

9. O. K. Sil'chenko, Astron. J. 117, 2725 (1999).

10. O. K. Sil'chenko, Astron. J. 118, 186 (1999).

11. V. V. Vlasyuk and O. K. Sil'chenko, Astron. Astrophys. 354, 28 (2000).

12. T. Wiklind, F. Combes, C. Henkel, and F. Wyrowski, Astron. Astrophys. 323, 727 (1997).

13. O. K. Sil'chenko, Astron. Zh. 71, 706 (1994) [Astron. Rep. 38, 624 (1994)].

14. R. Buta and K. L. Williams, Astron. J. 109, 543 (1995).

15. P. Chamaraux, C. Balkowski, and P. Fontanelli, Astron. Astrophys. 165, 15 (1986).

16. P. Prugniel and F. Simien, Astron. Astrophys. 282, L1 (1994).

17. V. L. Afanas'ev, V. V. Vlasyuk, S. N. Dodonov, and O. K. Sil'chenko, Preprint No. 54, SAO AN SSSR (1990).

18. V. V. Vlasyuk, Astrofiz. Issled. (Izv. SAO RAN) 36, 107 (1993).

19. G. Worthey, S. M. Faber, J. J. González, and D. Burstein, Astrophys. J., Suppl. Ser. 94, 687 (1994).

20. G. Worthey, Astrophys. J., Suppl. Ser. 95, 107 (1994).

21. R. Tantalo, C. Chiosi, and A. Bressan, Astron. Astrophys. 333, 419 (1998).

22. P. Poulain, Astron. Astrophys., Suppl. Ser. 72, 215 (1988).

23. A. U. Landolt, Astron. J. 104, 340 (1992).

24. S. C. Trager, G. Worthey, S. M. Faber, et al., Astrophys. J., Suppl. Ser. 116, 1 (1998).

25. O. K. Sil'chenko, Pis'ma Astron. Zh. 19, 693 (1993) [Astron. Lett. 19, 279 (1993)].

26. G. Worthey, S. M. Faber, and J. J. González, Astrophys. J. 398, 69 (1992). 
27. O. K. Sil'chenko, in Galaxies: The Third Dimension, Ed. by M. Rosado, L. Binette, and L. Arias (2002) (in press).

28. G. Magrelli, D. Bettoni, and G. Galetta, Mon. Not. R. Astron. Soc. 256, 500 (1992).

29. J. Kormendy, Astrophys. J. 217, 406 (1977).

30. W. E. Baggett, S. M. Baggett, and K. S. J. Anderson, Astron. J. 116, 1626 (1998).

31. J. Kormendy, Astrophys. J. 218, 333 (1977).

32. R. S. de Jong, Astron. Astrophys. 313, 45 (1996).
33. K. C. Freeman, Astrophys. J. 160, 811 (1970).

34. A. Vazdekis, E. Casuso, R. F. Peletier, and J. E. Beckman, Astrophys. J., Suppl. Ser. 106, 307 (1996).

35. O. K. Sil'chenko, V. V. Vlasyuk, and F. Alvarado, Astron. J. 121, 2499 (2001).

36. O. K. Sil'chenko and V. L. Afanasiev, Astron. Astrophys. 364, 479 (2000).

37. O. K. Sil'chenko and V. L. Afanasiev, Astron. Astrophys. 385, 1 (2002).

Translated by A. Dambis 